\title{
sciendo
}

Int. J. of Applied Mechanics and Engineering, 2020, vol.25, No.1, pp.177-197

DOI: $10.2478 /$ ijame-2020-0012

\section{TWO DIMENSIONAL DEFORMATION OF A MULTILAYERED THERMOELASTIC HALF-SPACE DUE TO SURFACE LOADS AND HEAT SOURCE}

\author{
A.K. VASHISHTH \\ Department of Mathematics, Kurukshetra University \\ Kurukshetra 136119, INDIA \\ E-mail: akvashishth@kuk.ac.in \\ K. RANI* \\ Department of Mathematics, Govt. College for Women \\ Bhodia Khera, Fatehabad 125050, INDIA \\ E-mail: karya4@gmail.com \\ K. SINGH \\ Department of Mathematics \\ Guru Jambheshwar University of Science and Technology \\ Hisar 125001, INDIA \\ E-mail: profkbgju@gmail.com
}

\begin{abstract}
This article deals with a 2-D problem of quasi-static deformation of a multilayered thermoelastic medium due to surface loads and heat source. The propagator matrix is obtained for the multilayered formalism of thermoelastic layers. Analytical solutions, in terms of the displacements, stresses, heat flux and temperature function, are obtained for normal strip and line loads, shear strip and line loads and strip and line heat sources. Numerical computation of the obtained analytical expressions is also done. The effects of layering have been studied. For the verification of the results, results of earlier studies have been obtained as particular cases of the present study.
\end{abstract}

Key words: multilayered half-space, thermoelastic, propagator matrix, surface loads, heat source.

\section{Introduction}

Multilayered structures exist in the nature frequently and are relevant to numerous engineering and geophysical problems (Yang et al. [1]). The Earth's structure may be considered as a multilayered halfspace. In addition to the above, there are various manmade materials or structures that can be regarded as multilayered structures for modelling applications in different fields, viz. civil, mechanical and biomechanical engineering, microelectronics and optics. The multilayered structure is assumed to be composed of homogeneous parallel layers.

Multilayered Earth's model has been considered by many researchers while studying the source problems. Singh [2] studied 3D problems of the static deformations of a multilayered elastic medium containing internal dislocation sources. Bache and Harkrider [3] considered the Earth as a layered model and formulated the theory of body waves due to a general seismic source. To investigate the two and three dimensional static deformations of an elastic medium, due to various surface loads (strip, circular and rectangular surface loads), Small and Booker [4] applied the finite layer method. Singh and Garg [6] studied two dimensional elastic dislocations in an isotropic multilayered half-space. Garg and Singh [7] studied two

\footnotetext{
* To whom correspondence should be addressed
} 
dimensional static deformation of a transversely isotropic multilayered half-space due to surface loads. Pan [8] considered a transversely isotropic and layered half-space to investigate its static deformation by general surface loads and dislocation sources. Garg et al. [10] discussed the static deformation of a stratified elastic half-space due to two dimensional surface loads. Pan et al. [12] developed an algorithm to calculate surface deformation due to circular loading on the surface of a layered elastic half-space. Recently, Ai et al. [13] and Zhang et al. [14] studied the deformation of a multilayered transversely isotropic elastic half space subjected to axisymmetric and nonaxisymmetric loading. Ai et al. [15] investigated the transient response of a transversely isotropic multilayered elastic half-space due to vertical loading.

The theory of thermoelasticity studies the effect of temperature on the distribution of stress and strain and the inverse effect of elastic change on temperature distribution. The thermoelastic parameters inside the Earth are generally functions of depth Dziewonski and Anderson [16]. Rundle [17] and Small and Booker [18] considered the Earth as an isotropic, horizontally layered thermoelastic half-space and studied the deformation caused by a decaying heat source. Using equations of quasi-static thermoelasticity, Pan [19] studied the transient deformation in a transversely isotropic and layered thermoelastic half-space by internal sources and surface loads. The analytical solutions in the form of displacements and stresses in multi-layered thermoelastic media due to varying temperature and concentrated loads were determined by Ghosh and Kanoria [20]. A number of problems related to the analysis of heat sources in multilayered thermoelastic media have been studied by Kolyano et al. [21], Jane and Lee [22], Lee et al. [23], Lee [24], Ai et al. [25], Ai and Wang [26], Ai et al.[27], Wang and Ai [28] etc. Hou et al. [29] derived two dimensional general and fundamental solutions for a line heat source acting on the surface of a semi-infinite orthotropic thermoelastic medium. Hou et al. [30] derived three-dimensional Green's function for a point heat source acting on the surface of a coated isotropic thermoelastic material. Ai and Wang [31] discussed thermal consolidation of a multilayered porous thermoelastic medium due to heat sources. Yang et al. [1] studied three dimensional axisymmetric problems for static response of multilayered thermoelastic media subjected to surface loads and containing heat source. The vector surface harmonics and propagator matrix method was used to investigate the behaviour of repository for heat-emitting high-level nuclear waste (decaying with time point heat source) in a geological formation. Ai et al. [32] introduced an extended precise integration solution for the coupled thermoelastic problems with the integral transformation techniques to illustrate the influence of the coupling of thermoelasticity and layered characteristics on the thermo-mechanical response. Ai et al. [33] discussed the thermo-mechanical response of a layered isotropic medium around a cylindrical heat source. $\mathrm{Lu}$ et al. [34] derived a modified scaled boundary finite element method to study three dimensional steadystate heat conduction in anisotropic layered media.

Although, a number of source problems involving multilayered thermoelastic media have been studied but mainly for heat source and are based on the uncoupled theory of thermoelasticity, which considers that the determination of the temperature field is independent of the stress and displacement fields. The coupled theory of thermoelasticity has not been seen taken into account to study mechanical source problem as it is appropriate to consider a thermoelastic medium for a realistic Earth's model.

In this paper, a plane strain problem of quasi-static deformation of a multilayered thermoelastic medium due to seismic sources and heat sources is studied. The layered thermoelastic medium consists of layers over a half-space. Surface loads and heat source are taken as sources. The basic equations of a coupled theory of thermoelasticity are used in formulation and propagator matrix method is used to analyze surface loading and temperature-induced deformations. Analytical solutions have been obtained in the form of integrals for normal strip loading, normal line loading, shear strip and line loading, strip heat source and line heat source. Numerical computation for displacements and heat flux for these sources is also done.

\section{Formulation of the problem}

A $p$ layered thermoelastic model, consisting of $p-1$ thermoelastic layers over the thermoelastic half-space is considered, as shown in Fig. 1 . Let $d_{n}$ denotes the thickness of the general $n$th layer bounded by the interfaces $z_{n-1}$ and $z_{n}$ and $H$ be the depth of the last interface. So 


$$
d_{n}=z_{n}-z_{n-1} \quad \text { and } \quad H=\sum_{n=1}^{p-1} d_{n}
$$

For a plane strain problem in the $y z$ - plane, the displacement vector is taken as

$$
\boldsymbol{u}=\boldsymbol{u}(y, z, t)=\left(u_{y}, u_{z}\right)
$$

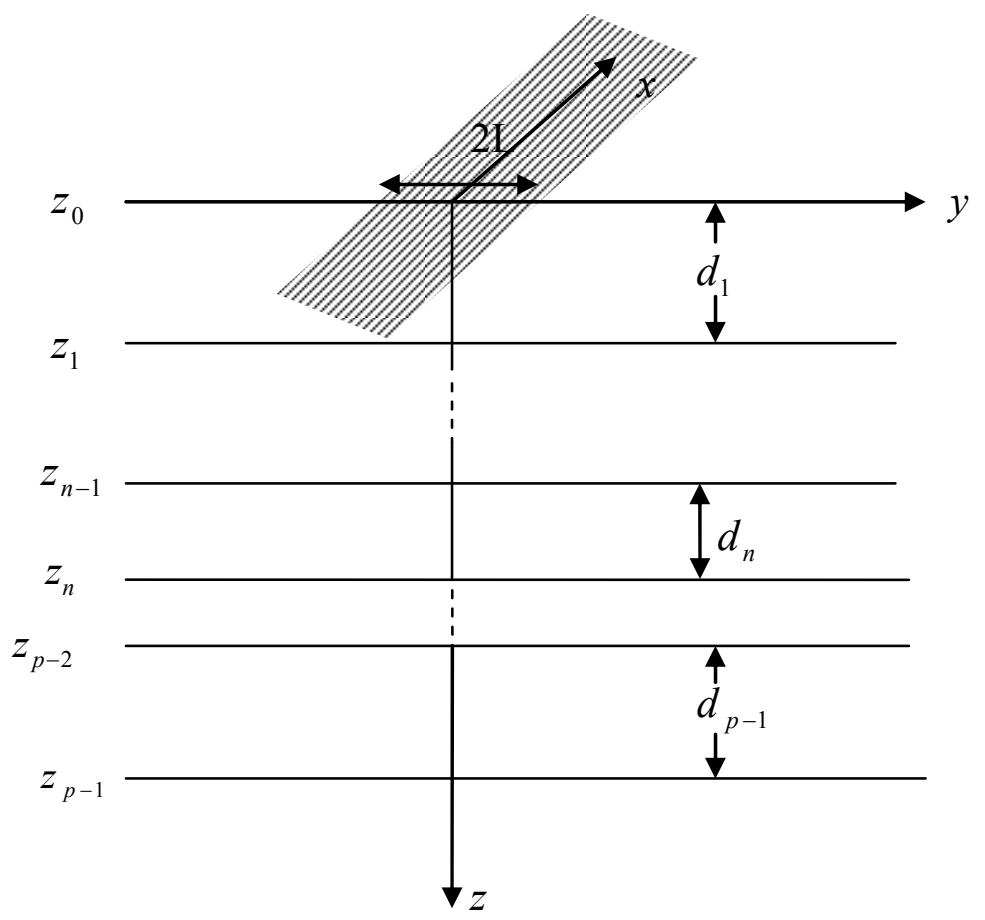

Fig.1. Multilayered thermoelastic half-space.

First, a strip of width $2 L$ and of infinite length in the $x$ - direction $(-L \leq y \leq L)$ over the surface $z_{0}(z=0)$ of multilayered half-space is considered and a normal force $\sigma_{0}$ acting uniformly on this strip is also considered. Next, normal and shear line forces and heat source are considered, respectively.

\section{Basic equations and their solutions}

The Duhamel-Neumann relations (stress-strain relations) for a homogeneous isotropic thermoelastic medium (Nowacki [35]) are

$$
\sigma=2 \mu \varepsilon+(\lambda \varepsilon-\beta \theta) I
$$

where $\sigma=\left[\begin{array}{cc}\sigma_{y y} & \sigma_{y z} \\ \sigma_{y z} & \sigma_{z z}\end{array}\right]$ and $\boldsymbol{\varepsilon}=\left[\begin{array}{cc}\varepsilon_{y y} & \varepsilon_{y z} \\ \varepsilon_{y z} & \varepsilon_{z z}\end{array}\right]=\frac{1}{2}\left(\nabla \boldsymbol{u}+\nabla^{T} \boldsymbol{u}\right)$ are stress and strain tensors, respectively; $\lambda$ and $\mu$ are Lame's constants; $\theta$ is the temperature deviation from the reference temperature $T_{0}$; 
$\beta=\alpha_{t}(3 \lambda+2 \mu) ; \alpha_{t}$ is the coefficient of linear thermal expansion; $\varepsilon=u_{y, y}+u_{z, z} ; \nabla \equiv\left[\frac{\partial}{\partial y} \frac{\partial}{\partial z}\right]$ and $\boldsymbol{I}=\left[\begin{array}{ll}1 & 0 \\ 0 & 1\end{array}\right]$

From Eq.(3.1), the strains can be obtained as

$$
\begin{aligned}
& 2 \mu \varepsilon_{y y}=(1-v) \sigma_{y y}-v \sigma_{z z}+\alpha_{0} \theta, \\
& 2 \mu \varepsilon_{z z}=(1-v) \sigma_{z z}-v \sigma_{y y}+\alpha_{0} \theta, \\
& 2 \mu \varepsilon_{y z}=\sigma_{y z}
\end{aligned}
$$

where $\alpha_{0}=2 \mu(1+v) \alpha_{t}$ and $v$ is Poisson's ratio.

The governing equations for a thermoelastic medium (Vashisth et al. [35]) are:

(i) Equations of equilibrium

$$
\nabla . \sigma=0
$$

(ii) Compatibility equation

$$
\varepsilon_{y y, z z}+\varepsilon_{z z, y y}=2 \varepsilon_{y z, y z}
$$

(iii) The equation of heat conduction

$$
\lambda_{0} \nabla^{2} \theta-\rho C_{e} \dot{\theta}-\beta T_{0} \dot{\varepsilon}=0
$$

where, $\lambda_{0}$ is the thermal conductivity, $\rho$ is the density, $C_{e}$ is the specific heat and superposed dot denotes derivative with respect to $t$.

Using Eqs (3.2) and (3.3) in Eq. (3.4), we get

$$
\nabla^{2}\left(\sigma_{y y}+\sigma_{z z}+2 \eta \theta\right)=0
$$

where

$$
\eta=\frac{\alpha_{0}}{2(1-v)}, \quad \nabla^{2} \equiv \nabla \cdot \nabla
$$

Let $U$ be the Airy's stress function. So, the stresses can be expressed as

$$
\sigma_{y y}=\frac{\partial^{2} U}{\partial z^{2}}, \quad \sigma_{z z}=\frac{\partial^{2} U}{\partial y^{2}}, \quad \sigma_{y z}=-\frac{\partial^{2} U}{\partial y \partial z}
$$

Using Eqs (3.2) and (3.7) in Eqs (3.5) and (3.6), we get

$$
\nabla^{2}\left(\nabla^{2} U+2 \eta \theta\right)=0,
$$




$$
\lambda_{0} \nabla^{2} \theta-\left(\rho C_{e}+\frac{\alpha_{0}^{2} T_{0}}{\mu(1-2 v)}\right) \dot{\theta}-\frac{\alpha_{0} T_{0}}{2 \mu}\left(\nabla^{2} \dot{U}\right)=0
$$

Applying the Laplace transform, Eqs (3.8)-(3.9) become

$$
\begin{aligned}
& \left(\nabla^{2}-\frac{s}{c}\right) \nabla^{2} \bar{\theta}=0, \\
& \left(\nabla^{2}-\frac{s}{c}\right) \nabla^{4} \bar{U}=0, \\
& c=\lambda_{0}\left[\rho C_{e}+\frac{\alpha_{0}{ }^{2} T_{0}}{\mu(1-2 v)}-\frac{\alpha_{0}{ }^{2} T_{0}}{2 \mu(1-v)}\right]^{-1} .
\end{aligned}
$$

where $\quad c=\lambda_{0}\left[\rho C_{e}+\frac{\alpha_{0}{ }^{2} T_{0}}{\mu(1-2 v)}-\frac{\alpha_{0}{ }^{2} T_{0}}{2 \mu(1-v)}\right]^{-1}$.

The bar over the symbols, which denotes the Laplace transform of the variable, is dropped hereafter for convenience.

Applying the Fourier sine/cosine transform to Eqs (3.10)-(3.11), solving the resulting differential equations and then taking inversion of the Fourier sine/cosine transform and simplifying further, we obtain

$$
\begin{aligned}
& \theta=\int_{0}^{\infty} R\left(\begin{array}{l}
\sin k y \\
\cos k y
\end{array}\right) d k, \\
& U=\int_{0}^{\infty} F\left(\begin{array}{l}
\sin k y \\
\cos k y
\end{array}\right) d k
\end{aligned}
$$

where

$$
\begin{aligned}
& R=\frac{s}{\gamma_{1}} \xi\left(L_{1} e^{-m z}+M_{1} e^{m z}\right)+\xi k^{2}\left(L_{3} e^{-k z}-M_{3} e^{k z}\right), \\
& F=L_{1} e^{-m z}+M_{1} e^{m z}+\left(L_{2}+L_{3} k z\right) e^{-k z}+\left(M_{2}+M_{3} k z\right) e^{k z} \\
& m=\left(k^{2}+s / c\right)^{1 / 2}, \quad \xi=\frac{2\left(v_{a}-v\right)}{\alpha_{0}}, \quad \gamma_{1}=-2 \eta c \xi, \quad v_{a}=v+\frac{\alpha_{0}^{2} T_{0}}{2 \mu}\left(\rho C_{e}+\frac{\alpha_{0} T_{0}}{\mu(1-2 v)}\right)^{-1},
\end{aligned}
$$

and the coefficients $L_{i}, M_{i}(i=1,2,3)$ may be functions of $k$. The expression $\left(\begin{array}{l}\sin k y \\ \cos k y\end{array}\right)$ denotes either $\sin k y$ or cosky. The phrases 'upper solution' and 'lower solution' will refer to the upper and lower terms of this expression, respectively.

For the layers of finite depth, both positive and negative exponential terms are retained. For finiteness of solution in the half-space ( $p$-th layer), where $z \rightarrow \infty$, only negative exponential terms are retained.

The stresses can be obtained as 


$$
\sigma_{y y}=\int_{0}^{\infty} G\left(\begin{array}{c}
\sin k y \\
\cos k y
\end{array}\right) d k, \quad \sigma_{z z}=\int_{0}^{\infty} N\left(\begin{array}{l}
\sin k y \\
\cos k y
\end{array}\right) k d k, \quad \sigma_{y z}=\int_{0}^{\infty} S\left(\begin{array}{l}
\cos k y \\
-\sin k y
\end{array}\right) k d k,
$$

where

$$
\begin{aligned}
& G=m^{2}\left(L_{1} e^{-m z}+M_{1} e^{m z}\right)+k^{2}\left(L_{2} e^{-k z}+M_{2} e^{k z}\right)+k^{2}\left((k z-2) L_{3} e^{-k z}+(k z+2) M_{3} e^{k z}\right) \\
& N=-k\left(L_{1} e^{-m z}+M_{1} e^{m z}+\left(L_{2}+L_{3} k z\right) e^{-k z}+\left(M_{2}+M_{3} k z\right) e^{k z}\right) \\
& S=m\left(L_{1} e^{-m z}-M_{1} e^{m z}\right)+k\left(L_{2} e^{-k z}-M_{2} e^{k z}-L_{3}(1-k z) e^{-k z}-M_{3}(1+k z) e^{k z}\right)
\end{aligned}
$$

The displacement components can now be obtained as

$$
u_{y}=\int_{0}^{\infty} V\left(\begin{array}{c}
\cos k y \\
-\sin k y
\end{array}\right) d k, \quad u_{z}=\int_{0}^{\infty} W\left(\begin{array}{l}
\sin k y \\
\cos k y
\end{array}\right) d k
$$

where

$$
\begin{aligned}
& V=-\frac{k}{2 \mu}\left(L_{1} e^{-m z}+M_{1} e^{m z}+L_{2} e^{-k z}+M_{2} e^{k z}+\right. \\
& \left.+L_{3}\left(2 \mathrm{v}_{a}-2+k z\right) e^{-k z}-M_{3}\left(2 \mathrm{v}_{a}-2-k z\right) e^{k z}\right), \\
& W=\frac{1}{2 \mu}\left\{m\left(L_{1} e^{-m z}-M_{1} e^{m z}\right)+k\left(L_{2} e^{-k z}-M_{2} e^{k z}\right)+\right. \\
& \left.+L_{3}\left(1-2 \mathrm{v}_{a}+k z\right) k e^{-k z}-M_{3}\left(-1+2 \mathrm{v}_{a}+k z\right) k e^{k z}\right\} .
\end{aligned}
$$

The flux of heat in the $z$-direction, given by the generalized Fourier law, can be obtained as

$$
q_{z}=\int_{0}^{\infty} Q\left(\begin{array}{c}
\sin k y \\
\cos k y
\end{array}\right) d k
$$

where

$$
Q=\lambda_{0} \xi \frac{m s}{\gamma_{1}}\left(L_{1} e^{-m z}-M_{1} e^{m z}\right)+\lambda_{0} \xi k^{3}\left(L_{3} e^{-k z}+M_{3} e^{k z}\right)
$$

The functions $V, W, S, N, R, Q$ are related through

where

$$
\Phi(z)=\boldsymbol{Z}(z) \boldsymbol{E}(z) \boldsymbol{A}
$$

$$
\begin{aligned}
& \boldsymbol{\Phi}(z)=\left[\begin{array}{llllll}
V & W & S & N & R & Q
\end{array}\right]^{T}, \quad \boldsymbol{A}=\left[\begin{array}{llllll}
L_{1} & M_{1} & L_{2} & M_{2} & L_{3} & M_{3}
\end{array}\right]^{T}, \\
& \boldsymbol{Z}(z)=\left[\begin{array}{ll}
\boldsymbol{a}(z) & \boldsymbol{b}(z) \\
\boldsymbol{c}(z) & \boldsymbol{d}(z)
\end{array}\right],
\end{aligned}
$$




$$
\begin{gathered}
\boldsymbol{a}(z)=\left[\begin{array}{cc}
-\frac{k}{2 \mu} & -\frac{k}{2 \mu} \\
\frac{m}{2 \mu} & -\frac{m}{2 \mu} \\
m & -m \\
-k & -k
\end{array}\right], \quad \boldsymbol{b}(z)=\left[\begin{array}{cccc}
-\frac{k}{2 \mu} & -\frac{k}{2 \mu} & -\frac{k}{2 \mu}\left(2 \mathrm{v}_{a}-2+k z\right) & -\frac{k}{2 \mu}\left(-2 \mathrm{v}_{a}+2+k z\right) \\
\frac{k}{2 \mu} & -\frac{k}{2 \mu} & \frac{k}{2 \mu}\left(1-2 \mathrm{v}_{a}+k z\right) & \frac{k}{2 \mu}\left(1-2 \mathrm{v}_{a}-k z\right) \\
k & -k & k(k z-1) & -k(k z+1) \\
-k & -k & -k^{2} z & -k^{2} z
\end{array}\right], \\
\boldsymbol{c}(z)=\left[\begin{array}{cc}
\frac{s}{\gamma_{1}} \xi & \frac{s}{\gamma_{1}} \xi \\
\frac{m s}{\gamma_{1}} \xi \lambda_{0} & -\frac{m s}{\gamma_{1}} \xi \lambda_{0}
\end{array}\right], \quad \boldsymbol{d}(z)=\left[\begin{array}{cccc}
0 & 0 & \xi k^{2} & -\xi k^{2} \\
0 & 0 & \xi \lambda_{0} k^{3} & \xi \lambda_{0} k^{3}
\end{array}\right] .
\end{gathered}
$$

\section{Equations for multilayered half-space}

The equations, described in Section 3, can now be written for the $n$-th layer by affixing a subscript ' $n$ '.

$$
\begin{aligned}
& \therefore \boldsymbol{\Phi}_{n}=\left[\begin{array}{llllll}
V_{n} & W_{n} & S_{n} & N_{n} & R_{n} & Q_{n}
\end{array}\right]^{T}=\boldsymbol{Z}_{n}(z) \boldsymbol{E}_{n}(z) \boldsymbol{A}_{n}, \quad\left(z_{n-1} \leq z \leq z_{n}\right) \\
& \Rightarrow \boldsymbol{A}_{n}=\boldsymbol{E}_{n}^{-1}\left(z_{n}\right) \boldsymbol{Z}_{n}^{-1}\left(z_{n}\right) \boldsymbol{\Phi}_{n}\left(z_{n}\right) .
\end{aligned}
$$

The continuity of displacements, stresses, temperature function and heat flux at the interfaces leads to

$$
\Phi_{n-1}\left(z_{n-1}\right)=\boldsymbol{P}_{n} \Phi_{n}\left(z_{n}\right)
$$

where $\boldsymbol{P}_{n}$ is called the propagator matrix and is

$$
\boldsymbol{P}_{n}=\boldsymbol{Z}_{n}\left(z_{n-1}\right) \boldsymbol{E}_{n}\left(z_{n-1}\right) \boldsymbol{E}_{n}^{-1}\left(z_{n}\right) \boldsymbol{Z}_{n}^{-1}\left(z_{n}\right)
$$

The elements of the matrix $\boldsymbol{P}_{n}$ are given in the Appendix A.

Repeated use of Eq.(4.3) gives

$$
\Phi_{l}(0)=D \Phi_{p}(H)
$$

where

$$
\boldsymbol{D}=\boldsymbol{P}_{1} \boldsymbol{P}_{2} \boldsymbol{P}_{3} \ldots \ldots . \boldsymbol{P}_{p-1}
$$

In the $\mathrm{p}$ - th layer, i.e. in the half-space, the solutions must be finite as $z \rightarrow \infty$ which requires

$$
M_{1 p}=M_{2 p}=M_{3 p}=0
$$

Equations (4.1), (4.5) and (4.7) imply that

$$
\left[\begin{array}{llllll}
V_{0} & W_{0} & S_{0} & N_{0} & R_{0} & Q_{0}
\end{array}\right]^{T}=\boldsymbol{B}\left[\begin{array}{llllll}
L_{1 p} & 0 & L_{2 p} & 0 & L_{3 p} & 0
\end{array}\right]^{T}
$$

where 


$$
\boldsymbol{B}=\boldsymbol{D} \boldsymbol{Z}_{p}(H) \boldsymbol{E}_{p}(H)
$$

For given $S_{0}, N_{0}$ and $R_{0}$, the solution of Eq.(4.8) can be written as

$$
\begin{aligned}
& V_{0}=\frac{1}{\Delta}\left(B_{11} \Delta_{1}+B_{13} \Delta_{2}+B_{15} \Delta_{3}\right), \quad W_{0}=\frac{1}{\Delta}\left(B_{21} \Delta_{1}+B_{23} \Delta_{2}+B_{25} \Delta_{3}\right), \\
& Q_{0}=\frac{1}{\Delta}\left(B_{61} \Delta_{1}+B_{63} \Delta_{2}+B_{65} \Delta_{3}\right)
\end{aligned}
$$

where

$$
\begin{aligned}
& \Delta=\operatorname{det}\left(C_{1}, C_{2}, C_{3}\right), \Delta_{1}=\operatorname{det}\left(C_{4}, C_{2}, C_{3}\right), \Delta_{2}=\operatorname{det}\left(C_{1}, C_{4}, C_{3}\right), \Delta_{3}=\operatorname{det}\left(C_{1}, C_{2}, C_{4}\right) \text { and } \\
& C_{1}=\left[\begin{array}{lll}
B_{31} & B_{41} & B_{51}
\end{array}\right]^{T}, C_{2}=\left[\begin{array}{llll}
B_{33} & B_{43} & B_{53}
\end{array}\right]^{T}, \\
& C_{3}=\left[\begin{array}{lll}
B_{35} & B_{45} & B_{55}
\end{array}\right]^{T}, C_{4}=\left[\begin{array}{lll}
S_{0} & N_{0} & R_{0}
\end{array}\right]^{T} .
\end{aligned}
$$

Using Eq.(4.10) in Eqs (3.20) and (3.23), $u_{y}(0), u_{z}(0)$ and $q_{z}(0)$ can be obtained.

For given $S_{0}, N_{0}$ and $Q_{0} ; V_{0}, W_{0}$ and $R_{0}$ can be obtained by replacing $B_{61}, B_{63}, B_{65}$ and $Q_{0}$ with $B_{51}, B_{53}, B_{55}$ and $R_{0}$ respectively in Eqs (4.10) and using these in Eqs (3.20) and (3.12); $u_{y}(0), u_{z}(0)$ and $\theta(0)$ can be obtained.

\section{Boundary conditions}

\subsection{Surface loads}

Let us assume the surface $z=0$ as isothermal surface. Hence $\theta=0$ at $z=0$. The stresses, for different type of prescribed loads over the surface, can be written as:

5.1.1 Normal strip loading: $\sigma_{y z}=0, \quad \sigma_{z z}=\left\{\begin{array}{ll}0 & \text { for }|y|>L \\ -\frac{\sigma_{0}}{2 L} & \text { for }|y|<L\end{array} \quad\right.$ at $\quad z=0$.

5.1.2 Normal line loading: $\sigma_{y z}=0, \quad \sigma_{z z}=-\sigma_{0} \delta(y), \quad$ at $z=0$.

5.1.3 Shear strip loading: $\sigma_{z z}=0, \quad \sigma_{y z}=\left\{\begin{array}{ll}0 & \text { for }|y|>L \\ -\frac{\tau_{0}}{2 L} & \text { for }|y|<L\end{array}\right.$ at $z=0$

where $\tau_{0}$ is the force intensity applied in the positive $y$ direction.

5.1.4 Shear line loading: $\sigma_{z z}=0, \quad \sigma_{y z}=-\tau_{0} \delta(y), \quad$ at $\quad z=0$.

\subsection{Heat source}

For a prescribed heat source, $\theta$ is known. Let us consider a traction free surface $z=0$. Hence, $\sigma_{y z}=\sigma_{z z}=0$ at $z=0$. 
5.2.1 Strip heat source: $\theta=\left\{\begin{array}{ll}0 & \text { for }|y|>L \\ \frac{\theta_{0}}{2 L} & \text { for }|y|<L\end{array} \quad\right.$ at $\quad z=0$,

where $\theta_{0}$ is the heat source density.

5.2.2 Line heat source: $\theta=\theta_{0} \delta(y), \quad$ at $\quad z=0$.

Taking the Laplace and Fourier sine/cosine transform of Eqs (5.1)-(5.6), solving, taking inverse transform and then comparing the resulting equations with (3.12) and (3.16) and selecting appropriate 'Upper or Lower solution', we obtain values of $S_{0}, N_{0}$ and $R_{0}$ as given in Tab.1 for different type of loadings.

Table 1. Values of $S_{0}, N_{0}$ and $R_{0}$ for different type of loads on the surface.

\begin{tabular}{|l|l|l|l|l|l|l|}
\hline & $\begin{array}{l}\text { Normal strip } \\
\text { loading }\end{array}$ & $\begin{array}{l}\text { Normal line } \\
\text { loading }\end{array}$ & $\begin{array}{l}\text { shear strip } \\
\text { loading }\end{array}$ & $\begin{array}{l}\text { shear line } \\
\text { loading }\end{array}$ & $\begin{array}{l}\text { Strip heat } \\
\text { source }\end{array}$ & $\begin{array}{l}\text { Line heat } \\
\text { source }\end{array}$ \\
\hline$S_{0}$ & 0 & 0 & $-\frac{\tau_{0}}{\pi s k} \frac{\sin k L}{k L}$ & $-\frac{\tau_{0}}{\pi s k}$ & 0 & 0 \\
\hline$N_{0}$ & $-\frac{\sigma_{0}}{\pi k s} \frac{\sin k L}{k L}$ & $-\frac{\sigma_{0}}{\pi k s}$ & 0 & 0 & 0 & 0 \\
\hline$R_{0}$ & 0 & 0 & 0 & 0 & $\frac{\theta_{0}}{\pi s} \frac{\sin k L}{k L}$ & $\frac{\theta_{0}}{\pi s}$ \\
\hline $\begin{array}{l}\text { Upper solution } \\
\text { Lower solution }\end{array}$ & $\begin{array}{l}\text { Lower } \\
\text { solution }\end{array}$ & $\begin{array}{l}\text { Lower } \\
\text { solution }\end{array}$ & $\begin{array}{l}\text { Upper } \\
\text { solution }\end{array}$ & $\begin{array}{l}\text { Upper } \\
\text { solution }\end{array}$ & $\begin{array}{l}\text { Lower } \\
\text { solution }\end{array}$ & $\begin{array}{l}\text { Lower } \\
\text { solution }\end{array}$ \\
\hline
\end{tabular}

\section{Uniform half-space}

When the model contains only a thermoelastic half-space $(0 \leq z<\infty)$ and layers are absent, then Eq.(4.9) reduces to $\boldsymbol{B}=\boldsymbol{Z}(0)$.

For normal line loading, we have

$$
L_{1}=\frac{\sigma_{0}}{\pi s \Lambda}, \quad L_{2}=\frac{\sigma_{0}}{\pi s \Lambda k^{2}}\left(\frac{-s}{\gamma_{1}}-m k\right), \quad L_{3}=\frac{-\sigma_{0}}{\pi \gamma_{1} k^{2} \Lambda},
$$

where $\Lambda=\left(-\frac{s}{\gamma_{1}}-m k+k^{2}\right)$

Substituting these values in Eq. (4.8), we get $V_{0}, W_{0}$ and $Q_{0}$ as

$$
V_{0}=\frac{-\sigma_{0}}{2 \pi s \mu \Lambda}\left(k-m+\left(1-2 v_{a}\right) \frac{s}{k \gamma_{1}}\right), W_{0}=-\frac{\left(1-v_{a}\right) \sigma_{0}}{\pi \mu \gamma_{1} k \Lambda}, Q_{0}=-\frac{\sigma_{0} \lambda_{0}}{2 \pi \eta c \Lambda}(m-k)
$$

Substituting Eq. (6.2) in Eqs (3.20) and (3.23), the displacements and heat flux on the surface can be obtained. 
written as

The solutions in the interior of the half-space for normal line loading are also obtained and are

$$
\begin{aligned}
& u_{y}(y, z, s)=\frac{\sigma_{0}}{2 \pi \mu s} \int_{0}^{\infty}\left(k e^{-m z}-m e^{-k z}-\frac{s}{\gamma_{1} k}\left(2 v_{a}-1+k z\right) e^{-k z}\right) \frac{\sin k y}{\Lambda} d k, \\
& u_{z}(y, z, s)=\frac{\sigma_{0}}{2 \pi \mu s} \int_{0}^{\infty}\left(m\left(e^{-m z}-e^{-k z}\right)-\frac{s}{\gamma_{1} k}\left(2-2 v_{a}+k z\right) e^{-k z}\right) \frac{\cos k y}{\Lambda} d k, \\
& q_{z}(y, z, s)=-\frac{\sigma_{0} \lambda_{0}}{2 \pi \eta c} \int_{0}^{\infty}\left(m e^{-m z}-k e^{-k z}\right) \frac{\cos k y}{\Lambda} d k, \\
& \sigma_{z z}(y, z, s)=-\frac{\sigma_{0}}{\pi s} \int_{0}^{\infty}\left(k^{2} e^{-m z}-m k e^{-k z}-(1+k z) \frac{s}{\gamma_{1}} e^{-k z}\right) \frac{\cos k y}{\Lambda} d k, \\
& \sigma_{y z}(y, z, s)=-\frac{\sigma_{0}}{\pi s} \int_{0}^{\infty}\left(m k\left(e^{-m z}-e^{-k z}\right)-k z \frac{s}{\gamma_{1}} e^{-k z}\right) \frac{\sin k y}{\Lambda} d k, \\
& \theta(y, z, s)=-\frac{\sigma_{0}}{2 \pi \eta c} \int_{0}^{\infty}\left(e^{-m z}-e^{-k z}\right) \frac{\cos k y}{\Lambda} d k .
\end{aligned}
$$

If the deformation occurs so slowly that the heat has sufficient time to diffuse from the material elements, there will be no temperature change from the reference temperature. In this limit of long time deformation, the response is labeled isothermal and the stress strain relation will be as in a linear elastic solid. Hence $t \rightarrow \infty$ gives isothermal response.

So, taking the limit $t \rightarrow \infty$ and using the result that $\lim _{t \rightarrow \infty} f(t)=\lim _{s \rightarrow 0} s \bar{f}(s)$, and evaluating the integrals given in Eqs (6.3)-(6.8) analytically, we have $q_{z}=\theta=0$,

$$
\begin{aligned}
& u_{y}(y, z)=\frac{\sigma_{0}}{2 \pi \mu}\left[(2 v-1) \tan ^{-1}\left(\frac{y}{z}\right)+\frac{y z}{y^{2}+z^{2}}\right], \\
& u_{z}(y, z)=\frac{\sigma_{0}}{2 \pi \mu}\left[-(1-v) \ln \left(y^{2}+z^{2}\right)+\frac{z^{2}}{y^{2}+z^{2}}\right], \\
& \sigma_{z z}(y, z)=\frac{\sigma_{0}}{\pi}\left[\frac{-2 z^{3}}{\left(y^{2}+z^{2}\right)^{2}}\right], \quad \sigma_{y z}(y, z)=\frac{\sigma_{0}}{\pi}\left[\frac{-2 y z^{2}}{\left(y^{2}+z^{2}\right)^{2}}\right] .
\end{aligned}
$$

These results match with the corresponding results of elastic medium (Sneddon [37]; Garg and Singh [38]). 
For shear line loading, the solutions are obtained as

$$
\begin{aligned}
& u_{y}(y, z, s)=-\frac{\tau_{0}}{2 \pi \mu s} \int_{0}^{\infty}\left(k\left(e^{-m z}-e^{-k z}\right)-\frac{s}{\gamma_{1} k}\left(2 v_{a}-2+k z\right) e^{-k z}\right) \frac{\cos k y}{\Lambda} d k, \\
& u_{z}(y, z, s)=\frac{\tau_{0}}{2 \pi \mu s} \int_{0}^{\infty}\left(m e^{-m z}-k e^{-k z}-\frac{s}{\gamma_{1} k}\left(1-2 v_{a}+k z\right) e^{-k z}\right) \frac{\sin k y}{\Lambda} d k, \\
& q_{z}(y, z, s)=-\frac{\tau_{0} \lambda_{0}}{2 \pi \eta c} \int_{0}^{\infty}\left(m e^{-m z}-k e^{-k z}\right) \frac{\sin k y}{\Lambda} d k, \\
& \sigma_{z z}(y, z, s)=-\frac{\tau_{0}}{\pi s} \int_{0}^{\infty}\left(k^{2}\left(e^{-m z}-\left(1+z \frac{s}{k \gamma_{1}}\right) e^{-k z}\right) \frac{\sin k y}{\Lambda} d k,\right. \\
& \sigma_{y z}(y, z, s)=\frac{\tau_{0}}{\pi s} \int_{0}^{\infty}\left(m k e^{-m z}-k^{2} e^{-k z}+(1-k z) \frac{s}{\gamma_{1}} e^{-k z}\right) \frac{\cos k y}{\Lambda} d k \\
& \theta(y, z, s)=-\frac{\tau_{0}}{2 \pi \eta c} \int_{0}^{\infty}\left(e^{-m z}-e^{-k z}\right) \frac{\sin k y}{\Lambda} d k .
\end{aligned}
$$

Taking the limit $t \rightarrow \infty$ and evaluating the integrals (6.10)-(6.15) analytically, we have $q_{z}=\theta=0$,

$$
\begin{aligned}
& u_{y}(y, z)=\frac{\tau_{0}}{2 \pi \mu}\left[-(1-v) \ln \left(y^{2}+z^{2}\right)-\frac{z^{2}}{y^{2}+z^{2}}\right], \\
& u_{z}(y, z)=\frac{\tau_{0}}{2 \pi \mu}\left[(1-2 v) \tan ^{-1}\left(\frac{y}{z}\right)+\frac{y z}{y^{2}+z^{2}}\right], \\
& \sigma_{z z}(y, z)=\frac{\tau_{0}}{\pi}\left[\frac{-2 y z^{2}}{\left(y^{2}+z^{2}\right)^{2}}\right], \quad \sigma_{y z}(y, z)=\frac{\tau_{0}}{\pi}\left[\frac{-2 y^{2} z}{\left(y^{2}+z^{2}\right)^{2}}\right] .
\end{aligned}
$$

These results match with the corresponding results of elastic medium (Sneddon [37]; Garg and Singh [38]).

For line heat source, the solutions are obtained as

$$
u_{y}(y, z, s)=\frac{-\theta_{0}}{2 \pi \mu s \xi} \int_{0}^{\infty}\left(k e^{-m z}-\left(k-\left(k z+2 \mathrm{v}_{a}-2\right)(m-k)\right) e^{-k z}\right) \frac{\sin k y}{\Lambda} d k
$$




$$
\begin{aligned}
& u_{z}(y, z, s)=\frac{-\theta_{0}}{2 \pi \mu s \xi} \int_{0}^{\infty}\left(m e^{-m z}+\left(-k+\left(1-2 v_{a}+k z\right)(m-k)\right) e^{-k z}\right) \frac{\cos k y}{\Lambda} d k, \\
& q_{z}(y, z, s)=\frac{\lambda_{0} \theta_{0}}{\pi s} \int_{0}^{\infty}\left(k^{2}(k-m) e^{-k z}-\frac{m s}{\gamma_{1}} e^{-m z}\right) \frac{\cos k y}{\Lambda} d k, \\
& \sigma_{z z}(y, z, s)=\frac{\theta_{0}}{\pi s \xi} \int_{0}^{\infty}\left(e^{-m z}-(1+(k-m) z) e^{-k z}\right) \frac{k^{2} \cos k y}{\Lambda} d k, \\
& \sigma_{y z}(y, z, s)=\frac{\theta_{0}}{\pi s \xi} \int_{0}^{\infty}\left(m e^{-m z}-(k+(1-k z)(m-k)) e^{-k z}\right) \frac{k \sin k y}{\Lambda} d k, \\
& \theta(y, z, s)=\frac{\theta_{0}}{\pi s} \int_{0}^{\infty}\left(k(k-m) e^{-k z}-\frac{s}{\gamma_{1}} e^{-m z}\right) \frac{\cos k y}{\Lambda} d k .
\end{aligned}
$$

\section{Numerical results and discussion}

For the numerical computation of the results, a model of three layers overlying half-space of a thermoelastic solid is considered. The parameters for numerical computation of the model are taken from Ahrens [39], Aki and Richards [40] and are given in Tab.2. Thickness of the layers is taken in the ratio 2:3:5. The solutions at the surface $z=0$ for surface line loading and line heat source are computed numerically and are presented graphically.

Table 2. Parameters of thermoelastic layers and half-space.

\begin{tabular}{|l|l|l|l|l|l|l|}
\hline Material & $\mu\left(\mathrm{kg} \mathrm{m}^{-1} \mathrm{~s}^{-2}\right)$ & $\lambda\left(\mathrm{kg} \mathrm{m}^{-1} \mathrm{~s}^{-2}\right)$ & $\alpha_{t}\left(\mathrm{~K}^{-1}\right)$ & $\rho\left(\mathrm{kg} \mathrm{m}^{-3}\right)$ & $T_{0}(K)$ & $C_{e}\left(\mathrm{~m}^{2} K^{-1} \mathrm{~s}^{-2}\right)$ \\
\hline $\begin{array}{l}\text { Layer 1 } \\
(\mathrm{CaO})\end{array}$ & $6.6378 \times(10)^{10}$ & $8.75 \times(10)^{10}$ & $3.92 \times(10)^{-5}$ & 3460 & 1000 & 888 \\
\hline $\begin{array}{l}\text { Layer 2 } \\
(\mathrm{MgO})\end{array}$ & $6.96 \times(10)^{10}$ & $9.67 \times(10)^{10}$ & $4.26 \times(10)^{-5}$ & 3516 & 1000 & 1166 \\
\hline $\begin{array}{l}\text { Layer 3 } \\
\text { Groussular } \\
\text { garnet) }\end{array}$ & $7.84 \times(10)^{10}$ & $10.92 \times(10)^{10}$ & $2.83 \times(10)^{-5}$ & 3542 & 1000 & 1041 \\
\hline $\begin{array}{l}\text { Half-space } \\
\text { Pyrope rich } \\
\text { garnet) }\end{array}$ & $8.51514 \times(10)^{10}$ & $11.4508 \times(10)^{10}$ & $3.11 \times(10)^{-5}$ & 3620 & 1000 & 1076 \\
\hline
\end{tabular}

The non-dimensional quantities are defined as

$$
Y=\frac{y}{H}, \quad Z=\frac{z}{H}, \quad T=\frac{2 c t}{H^{2}}, \quad U_{i}^{\prime}=\frac{\mu u_{i}}{\sigma_{0} H}, \quad \Theta=\frac{\eta \theta}{\sigma_{0}}, \quad \Sigma_{i j}=\frac{\sigma_{i j}}{\sigma_{0}}, \quad Q_{z}=\frac{\eta H q_{z}}{\sigma_{0} \lambda_{0}},
$$

for normal loading. For shear line loading and heat source, $\sigma_{0}$ is replaced by $\tau_{0}$ and $\eta \theta_{0}$, respectively. As the depth is made dimensionless, the coefficients in positive exponential terms in the solutions become less than one. So the check for convergent solutions is ensured and no numerical overflow is observed. 
For computation of the inverse Laplace transform, Schapery's [41] formula is used. Gauss quadrature formula has been used for the evaluation of results listed in Eqs (6.3)-(6.8), (6.10)-(6.15) and (6.17)-(6.22).
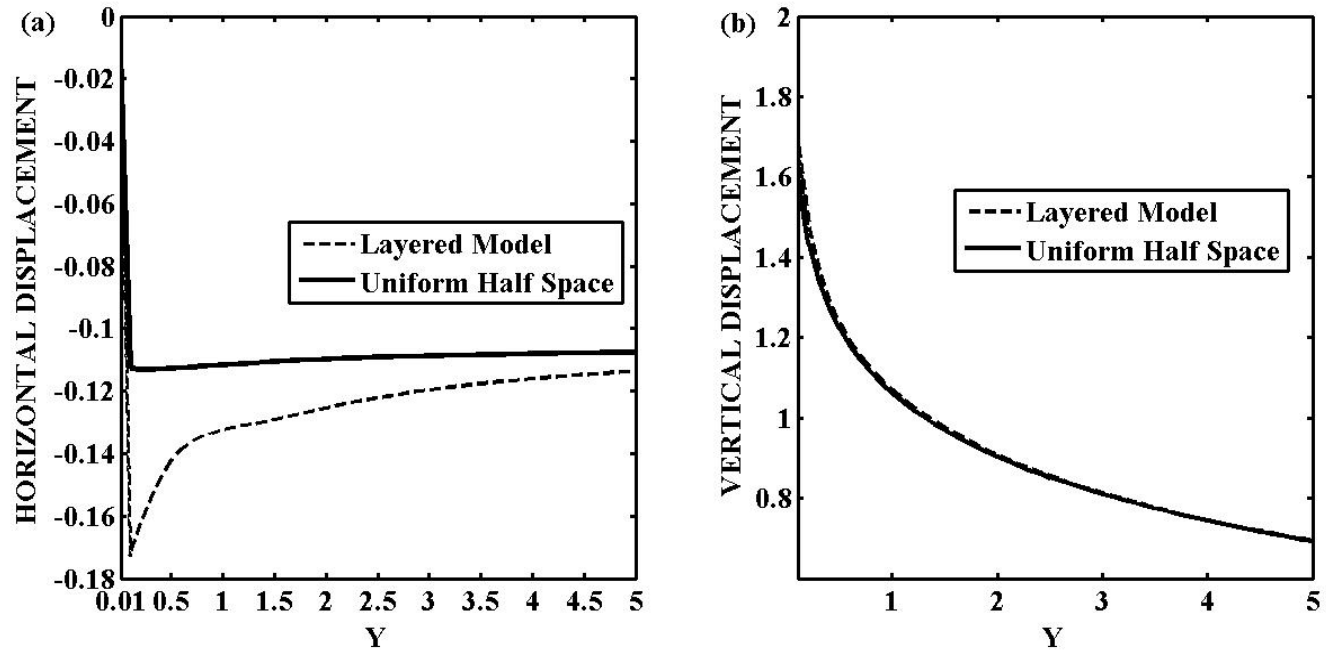

Fig.2. Variation of displacements along $Y$ at the surface, for normal line load; (a) $U_{y}^{\prime}$ (b) $U_{z}^{\prime}$.

For dimensionless time $T=1$, the variations of horizontal and vertical displacement components with the horizontal distance $(Y)$ at the surface $Z=0$ for normal line source are presented in Fig.2. The horizontal surface displacement increases gradually and the vertical displacement decreases slowly with the distance from the line source. The displacements have maximum magnitudes in the vicinity of $Y=0$. A comparison of the results of displacement components for a uniform half-space and layered model is also shown. It is observed that the magnitude of the displacements corresponding to layered model is much greater than that corresponding to the uniform half-space. Time history of displacement components for normal line loading is shown in Fig. 3 for $Y=1$. The horizontal surface displacement decreases steadily with time while the vertical displacement increases. The variation of horizontal displacement for layered model is greater than that of the uniform half-space.
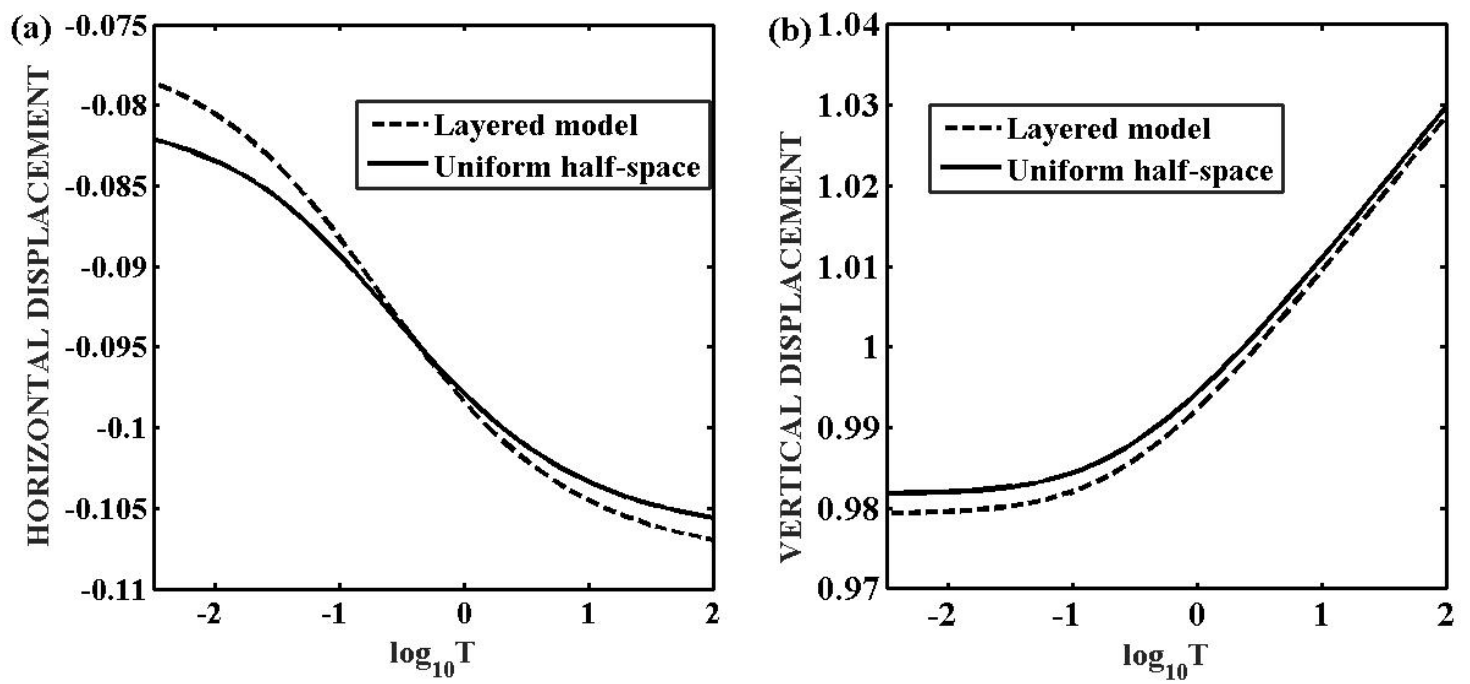

Fig.3. Variation of displacements with dimensionless time $T$ at the surface $Z=0$ for normal line load; (a) $U_{y}^{\prime}$ (b) $U_{z}^{\prime}$. 

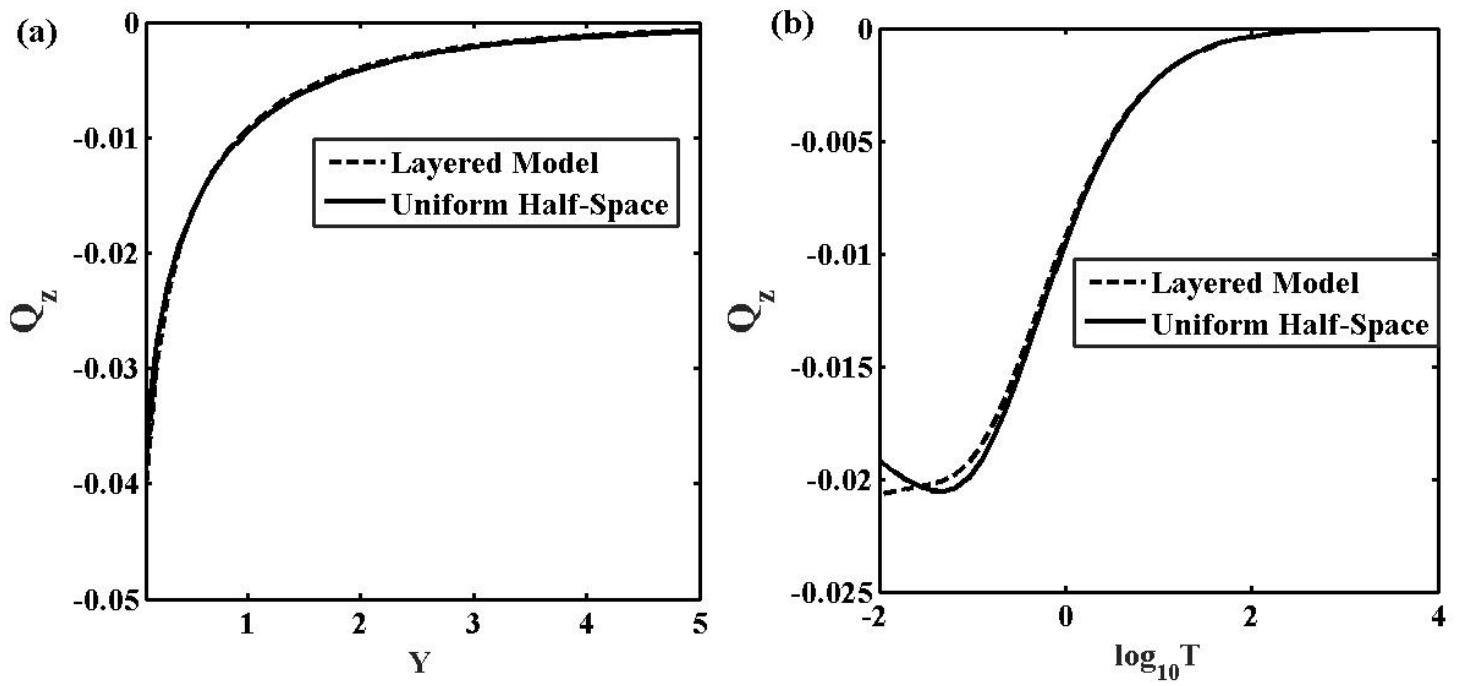

Fig.4. Surficial heat flux for normal line loading along (a) horizontal distance $Y$ (b) dimensionless time $T$.

Heat flux is computed for the normal line source for the half-space model and layered model and is plotted against the dimensionless horizontal distance $(Y)$ for $T=1$, as shown in Fig.4a. It increases first and then approaches zero as the distance increases. Time history of the heat flux for normal line loading is shown in Fig. $4 \mathrm{~b}$ for $Y=1$. The heat flux component firstly decreases then increases steadily and approaches zero as time increases. Initially, the difference between the layered model and the half-space model is significant. As the time elapses, the heat flux of the layered model approaches that for the half-space.

(a)

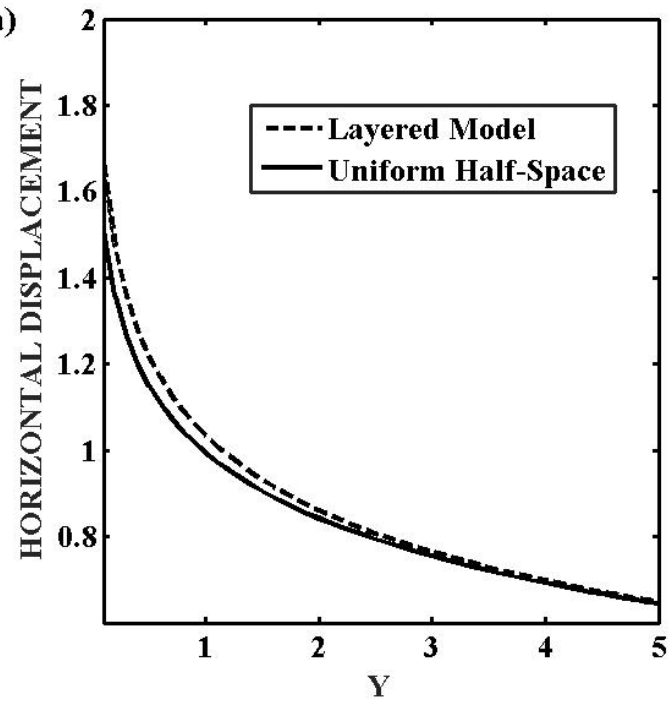

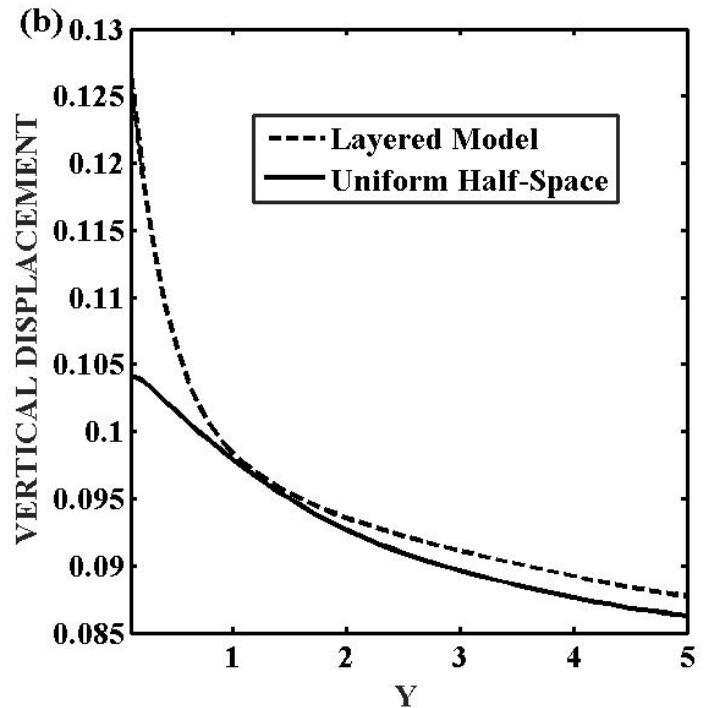

Fig.5. Variation of displacements with $Y$ at the surface $Z=0$ for shear line loading; (a) horizontal displacement (b) vertical displacement. 

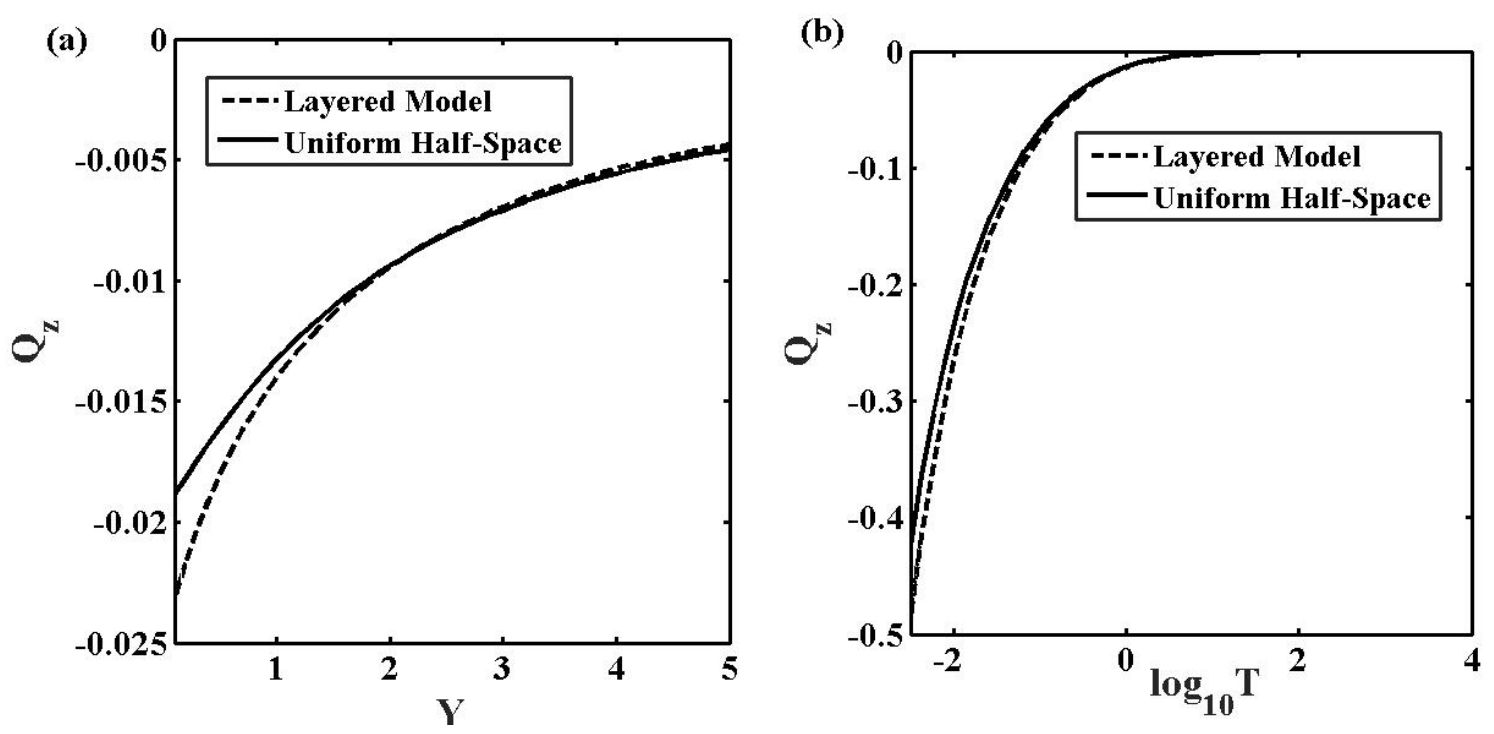

Fig.6. Heat flux for shear line loading at the surface $Z=0$ along (a) horizontal distance $Y$ (b) dimensionless time $T$.

Figure 5 exhibits the variation of the horizontal and vertical displacements with the horizontal distance $(Y)$ for shear line loading for $T=1$. It is observed that graph 5 a of horizontal displacement is same as the vertical displacement for normal line loading and graph $5 \mathrm{~b}$ of vertical displacement is the mirror image of horizontal displacement for normal line loading. This is also justified from the analytical expressions. It is observed that the displacement components in the layered model are more significant in the vicinity of the line source. Similar observations are for the graphs of time history of displacement components for shear line loading, so are not shown here.

The heat flux along horizontal distance $(Y)$ from line source for shear line loading is shown and compared for the layered model and half-space in Fig.6a. The heat flux steadily increases and approaches zero as the distance from the line source increases. Time history of heat flux for shear line loading is shown and is compared for the layered model and uniform half-space in Fig.6b. The heat flux increases smoothly with time and approaches zero as time increases. The comparison is significant when time is approaching zero. It signifies the importance of inclusion of thermoelastic effect in the source problems.
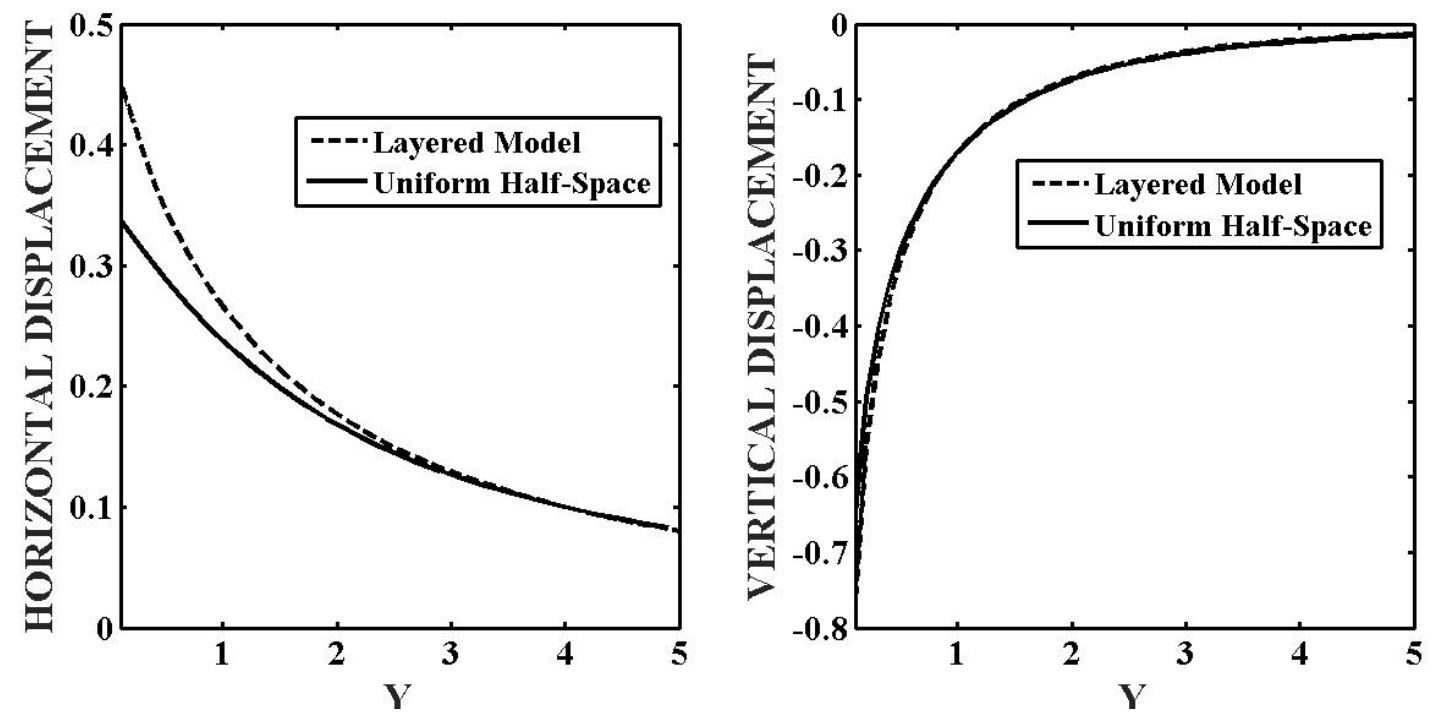

Fig.7. Variation of displacements at surface $Z=0$ with horizontal distance $Y$ due to line heat source; (a) $U_{y}^{\prime}$ (b) $U_{z}^{\prime}$. 

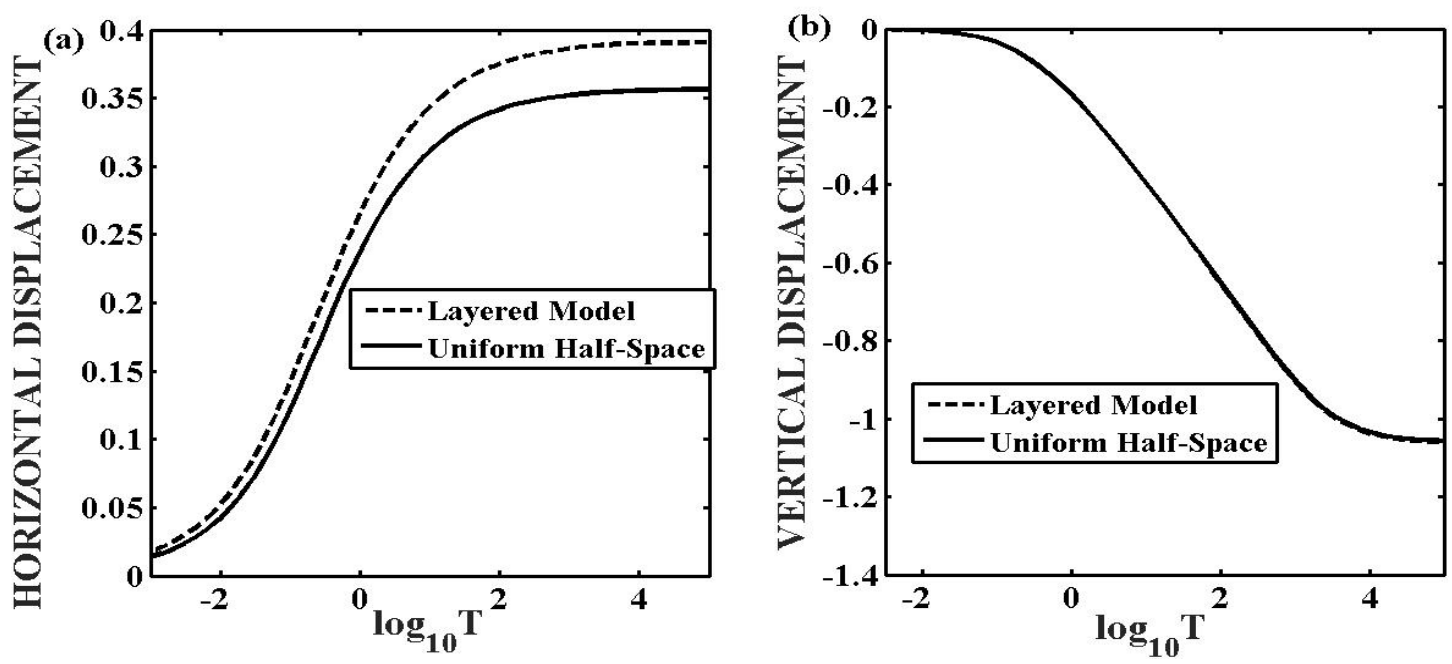

Fig.8. Variation of displacements with dimensionless time $T$ at surface $Z=0$ for line heat source; (a) $U_{y}^{\prime}$ (b) $U_{z}^{\prime}$.
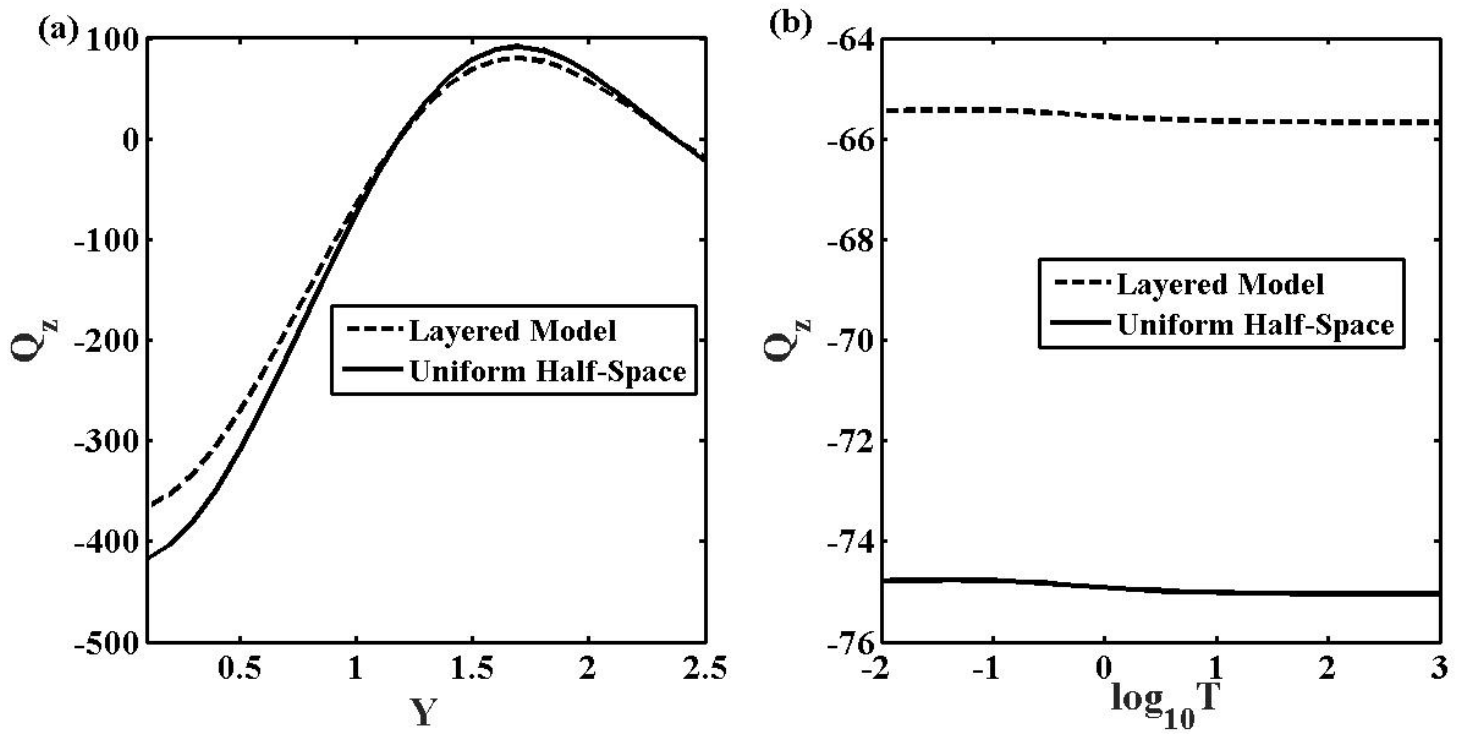

Fig.9. Surficial heat flux for line heat source along (a) horizontal distance $Y$ (b) dimensionless time $T$.

In Fig.7, horizontal displacement and vertical displacement are plotted along horizontal distance $(Y)$ from the line heat source at surface $Z=0$ for $T=1$ and are compared for the layered model and uniform halfspace. Along the horizontal distance $(Y)$ from the line heat source, the horizontal displacement decreases; the vertical displacement increases and both approach zero as the distance increases. Near the line heat source, the layered model has more deformation than the uniform half space. As the distance increases, both graphs coincide. For $Y=1$, the time history of displacements is presented in Fig.8. The horizontal displacement $U_{y}^{\prime}$ increases and the vertical displacement $U_{z}^{\prime}$ decreases with the dimensionless time $T$. It can be inferred from Fig. 7 and Fig. 8 that shear deformation is affected significantly by consideration of the layered model in the presence of heat source. 
Heat flux $\left(Q_{z}\right)$ is computed for the line heat source for the layered model and uniform half space and is plotted against the horizontal distance $(Y)$ as shown in Fig.9a. It sharply increases near the line of source, then decreases smoothly and approaches zero as the distance increases. In Fig.9b, the graph of heat flux with dimensionless time for the layered model and uniform half-space is plotted for $Y=1$. From Fig.9, it is observed that the increase in the number of layers decreases the magnitude of heat flux.

\section{Conclusion}

The present study deals with the two dimensional quasi-static deformation of a layered thermoelastic medium subjected to surface loads and heat source. The coupled theory of thermoelasticity is used for the governing equations. The multilayered problem is formulated using the propagator matrix method. Analytical solutions in the form of displacements, stresses and heat flux for normal and shear strip and line loading, strip and line heat source are derived for the uniform half space and multilayered model. Numerical results show that the number of layers influences the deformation of the medium as well as the flow of heat. The shear deformation is affected significantly by consideration of the layered model in the presence of normal line load and heat source and the normal deformation is affected significantly in the presence of shear line load. The effect of the layered model on heat flux due to surface loads and heat source is also considerable.

\section{Nomenclature}

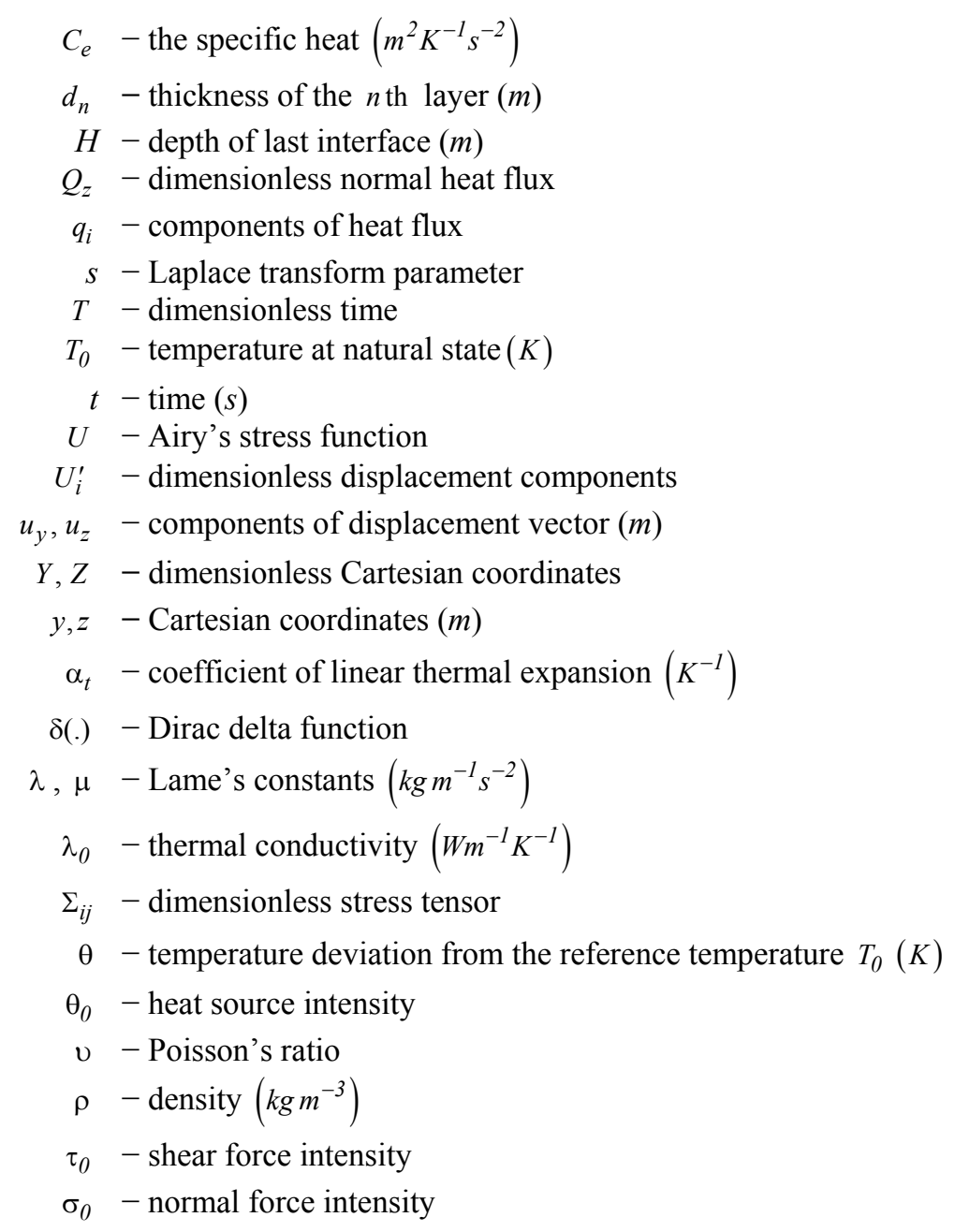


$\sigma_{i j} \quad$ components of stress tensor $\left(\mathrm{kg} \mathrm{m}^{-1} \mathrm{~s}^{-2}\right)$

$\Theta-$ dimensionless temperature difference function

\section{Appendix A}

The elements of $\boldsymbol{P}_{n}$ are given by

$$
\begin{aligned}
& P_{n}^{11}=\frac{2 \beta_{2} k^{2}}{s}\left(\cosh m d_{n}-\cosh k d_{n}\right)+\cosh k d_{n}+\beta_{1} k d_{n} \sinh k d_{n}=P_{n}^{33}, \\
& P_{n}^{12}=\frac{2 \beta_{2} k^{2}}{s}\left(\frac{k}{m} \sinh m d_{n}-\sinh k d_{n}\right)+2 \beta_{3} \sinh k d_{n}+\beta_{1} k d_{n} \cosh k d_{n}=-P_{n}^{43}, \\
& P_{n}^{13}=\frac{1}{2 \mu_{n}}\left(\frac{2 \beta_{2} k^{2}}{s}\left(-\frac{k}{m} \sinh m d_{n}+\sinh k d_{n}\right)+\left(\beta_{1}-2\right) \sinh k d_{n}-\beta_{1} k d_{n} \cosh k d_{n}\right), \\
& P_{n}^{14}=-\frac{1}{2 \mu_{n}}\left(\frac{2 \beta_{2} k^{2}}{s}\left(\cosh m d_{n}-\cosh k d_{n}\right)+\beta_{1} k d_{n} \sinh k d_{n}\right)=-P_{n}^{23}, \\
& P_{n}^{15}=\frac{k \eta_{n} c_{n}}{\mu_{n} s}\left(\cosh m d_{n}-\cosh k d_{n}\right), \quad P_{n}^{16}=\frac{\beta_{4}}{\mu_{n} s}\left(\frac{k}{m} \sinh m d_{n}-\sinh k d_{n}\right), \\
& P_{n}^{21}=\frac{2 \beta_{2} k}{s}\left(-m \sinh m d_{n}+k \sinh k d_{n}\right)+2 \beta_{3} \sinh k d_{n}-\beta_{1} k d_{n} \cosh k d_{n}=-P_{n}^{34}, \\
& P_{n}^{22}=\frac{2 \beta_{2} k^{2}}{s}\left(-\cosh m d_{n}+\cosh k d_{n}\right)+\cosh k d_{n}-\beta_{1} k d_{n} \sinh k d_{n}=P_{n}^{44}, \\
& P_{n}^{24}=\frac{1}{2 \mu_{n}}\left(\frac{2 \beta_{2} k}{s}\left(m \sinh m d_{n}-k \sinh k d_{n}\right)+\left(\beta_{1}-2\right) \sinh k d_{n}+\beta_{1} k d_{n} \cosh k d_{n}\right), \\
& P_{n}^{25}=\frac{\eta_{n} c_{n}}{\mu_{n} s}\left(-m \sinh m d_{n}+k \sinh k d_{n}\right), \quad P_{n}^{26}=\frac{\beta_{4}}{\mu_{n} s}\left(-\cosh m d_{n}+\cosh k d_{n}\right), \\
& P_{n}^{31}=-2 \mu_{n} \beta_{1}\left(\frac{\gamma_{1} k}{s}\left(m \sinh m d_{n}-k \sinh k d_{n}\right)+\sinh k d_{n}+k d_{n} \cosh k d_{n}\right), \\
& P_{n}^{32}=-2 \mu_{n} \beta_{1}\left(\frac{\gamma_{1} k^{2}}{s}\left(\cosh m d_{n}-\cosh k d_{n}\right)+k d_{n} \sinh k d_{n}\right)=-P_{n}^{41}, \quad P_{n}^{35}=2 \mu_{n} P_{n}^{25}, \quad P_{n}^{36}=2 \mu_{n} P_{n}^{26},
\end{aligned}
$$




$$
\begin{aligned}
& P_{n}^{42}=2 \mu_{n} \beta_{1}\left(\frac{\gamma_{1} k^{2}}{s}\left(\frac{k}{m} \sinh m d_{n}-\sinh k d_{n}\right)-\sinh k d_{n}+k d_{n} \cosh k d_{n}\right), P_{n}^{45}=2 \mu_{n} P_{n}^{15}, \\
& P_{n}^{46}=2 \mu_{n} P_{n}^{16}, \\
& P_{n}^{51}=2 \mu_{n} \xi_{n} \beta_{1} k\left(-\cosh m d_{n}+\cosh k d_{n}\right)=-2 \mu_{n} P_{n}^{54}, \\
& P_{n}^{52}=2 \mu_{n} \xi_{n} \beta_{1} k\left(-\frac{k}{m} \sinh m d_{n}+\sinh k d_{n}\right)=-2 \mu_{n} P_{n}^{53}, \quad P_{n}^{55}=\cosh m d_{n}=P_{n}^{66}, \\
& P_{n}^{56}=\frac{1}{m \lambda_{0 n}} \sinh m d_{n}, \quad P_{n}^{61}=2 T_{0 n} \eta_{n} c_{n} k^{2}\left(-\frac{m}{k} \sinh m d_{n}+\sinh k d_{n}\right)=-2 \mu_{n} P_{n}^{64}, \\
& P_{n}^{62}=2 T_{0 n} \eta_{n} c_{n} k^{2}\left(\cosh k d_{n}-\cosh m d_{n}\right)=-2 \mu_{n} P_{n}^{63}, \quad P_{n}^{65}=m \lambda_{0 n} \sinh m d_{n},
\end{aligned}
$$

where

$$
\beta_{1}=\frac{1}{2\left(1-v_{a n}\right)}, \quad \beta_{2}=\frac{c_{n}\left(v_{n}-v_{a n}\right)}{2\left(1-v_{n}\right)\left(1-v_{a n}\right)}=\frac{1}{2} \gamma_{1} \beta_{1}, \quad \beta_{3}=\frac{1}{2}\left(1-\beta_{1}\right), \quad \beta_{4}=\frac{c_{n} \eta_{n}}{\lambda_{0 n}}=\frac{\mu_{n} \xi_{n} \beta_{1}}{T_{0 n}}
$$

\section{References}

[1] Yang Y., Datcheva M. and Schanz T. (2016): Axisymmetric analysis of multilayered thermoelastic media with application to a repository for heat-emitting high-level nuclear waste in a geological formation. - Geophys. J. Int., vol.206, pp.1144-1161.

[2] Singh S.J. (1970): Static deformation of a multilayered half-space by internal sources. - J. Geophys. Res., vol.75, pp.3257-3263.

[3] Bache T.C. and Harkrider D.G. (1976): The body waves due to a general seismic source in a layered Earth model: 1 Formulation of the theory. - Bulletin of the Seismological Society of America, vol.66, No.6, pp.1805-1819.

[4] Small J.C. and Booker J.R. (1984): Finite layer analysis of layered elastic materials using a flexibility approach. Part 1-strip loadings. - International Journal for Numerical Methods in Engineering, vol.20, pp.1025-1037.

[5] Small J.C. and Booker J.R. (1986): Finite layer analysis of layered elastic materials using a flexibility approach. Part 2-circular and rectangular loadings. - Int. J. Numer. Methods Engg., vol.23, pp.959-978.

[6] Singh S.J. and Garg N.R. (1985): On two-dimensional elastic dislocations in a multilayered half-space. - Phys. Earth Planet. Inter., vol.40, pp.135-145.

[7] Garg N.R. and Singh S.J. (1987): 2-D static response of a transversely isotropic multilayered half-space to surface loads. - Indian J. Pure Appl. Math., vol.18, pp.763-777.

[8] Pan E. (1989): Static response of a transversely isotropic and layered half-space to general surface loads. - Phys. Earth Planet. Inter., vol.54, pp.353-363.

[9] Pan E. (1989): Static response of a transversely isotropic and layered half-space to general dislocation sources. Phys. Earth Planet. Inter., vol.58, pp.103-117.

[10] Garg N.R., Singh S.J. and Manchanda S. (1991): Static deformation of an orthotropic multilayered elastic halfspace by two-dimensional surface loads. - Proc. Indian Acad. Sci. (Earth Planet. Sci.), vol.100, No.2, pp.205-218.

[11] Garg N.R., Singh S.J. and Rani, S. (1992): Static deformation of a stratified medium by surface loads. - Indian J. Pure Math., vol.23, No.9, pp.675-692. 
[12] Pan E., Bevis M., Han F., Zhou H. and Zhu R. (2007): Surface deformation due to loading of a layered elastic half-space: a rapid numerical kernel based on a circular loading element. - Geophys. J. Int., vol.171, pp.11-24.

[13] Ai Z.Y., Feng D.L. and Cang N.R. (2014): Analytical layer element solutions for deformations of transversely isotropic multilayered elastic media under nonaxisymmetric loading. - Int. J. Numer. Anal. Meth. Geomech., vol.38, pp.1585-1599.

[14] Zhang P., Lin G., Liu J. and Wang W. (2016): Response of multilayered transversely isotropic medium due to axisymmetric loads. - Int. J. Numer. Anal. Meth. Geomech., vol.40, pp.827-864

[15] Ai Z.Y., Liu C.L. and Wang L.H. (2017): Transient response of a transversely isotropic multilayered half-space due to a vertical loading. - Applied Mathematical Modelling, vol.50, pp.414-431

[16] Dziewonski A.M. and Anderson D.L. (1981): Preliminary reference earth model. - Phys. Earth Planet. Inter., vol.25, No.4, pp.297-356.

[17] Rundle J.B. (1982): Some solutions for static and pseudo-static deformation in layered, nonisothermal, porous media. - J. Phys. Earth, vol.30, pp.421-440.

[18] Small J.C. and Booker J.R. (1986): The behaviour of layered soil or rock containing a decaying heat source. - Int. J. Numer. Anal. Methods Geomech., vol.10, pp.501-519.

[19] Pan E. (1990): Thermoelastic deformation of a transversely isotropic and layered half-space by surface loads and internal sources. - Phys. Earth Planet. Inter., vol.60, pp.254-264

[20] Ghosh M.K. and Kanoria M. (2007): Displacements and stresses in composite multi-layered media due to varying temperature and concentrated load. - Applied Mathematics and Mechanics (English Edition), vol.28, No.6, pp.811-822.

[21] Kolyano Y.M., Protsyuk B.V. and Sinyuta V.M. (1991): The axissymmetric static problem of thermoelasticity for a multilayered cylinder. - J. Appl. Maths. Mechs., vol.55, No.6, pp.920-926.

[22] Jane K.C. and Lee Z.Y. (1999): Thermoelasticity of multilayered cylinders. - Journal of Thermal Stresses, vol.22, pp.57-74.

[23] Lee Z.Y., Chen C.K. and Hung C.I. (2001): Transient thermal stress analysis of multilayered hollow cylinder. Acta Mechanica, vol.151, pp.75-88.

[24] Lee Z.Y. (2004): Coupled problem of thermoelasticity for multilayered spheres with time dependent boundary conditions. - Journal of Marine Science and Technology, vol.12, No.2, pp.93-101.

[25] Ai Z.Y., Wang L.J. and Li B. (2015): Analysis of axisymmetric thermo-elastic problem in multilayered material with anisotropic thermal diffusivity. - Computers and Geotechnics, vol.65, pp.80-86.

[26] Ai Z.Y. and Wang L.J. (2015): Time-dependent analysis of 3D thermo-mechanical behaviour of a layered halfspace with anisotropic thermal diffusivity. - Acta Mech., vol.226, No.9, pp.2939-2954

[27] Ai Z.Y., Wang L.U. and Zeng K. (2015): Analytical layer-element method for 3D thermoelastic problem of layered medium around a heat source. - Meccanica, vol.50, pp.49-59.

[28] Wang L.J. and Ai Z.Y. (2015): Plane strain and three-dimensional analyses for thermo-mechanical behavior of multilayered transversely isotropic materials. - International Journal of Mechanical Sciences, vol.103, pp.199211.

[29] Hou P.F., He S. and Chen C.P. (2011): 2D general solution and fundamental solution for orthotropic thermoelastic materials. - Engineering Analysis with Boundary Elements, vol.35, pp.56-60.

[30] Hou P.F., Jiang H.Y., Tong J. and Xiong S.M. (2014): Study on the coated isotropic thermoelastic material based on the three-dimensional Green's function for a point heat source. - International Journal of Mechanical Sciences, vol.83, pp.155-162.

[31] Ai Z.Y. and Wang L.J. (2016): Three-dimensional thermo-hydro-mechanical responses of stratified saturated porothermoelastic material. - Applied Mathematical Modelling, vol.40, pp.8912-8933.

[32] Ai Z.Y., Wang Q.L. and Wang L.J. (2016): Axisymmetric coupled thermo-mechanical response of multilayered elastic medium. - Meccanica, vol.51, pp.1405-1417. 
[33] Ai Z.Y., Zhao Z. and Wang L.J. (2017): Thermo-mechanical coupling response of a layered isotropic medium around a cylindrical heat source. - Computers and Geotechnics, vol.83, pp.159-167.

[34] Lu S., Liu J., Lin G. and Zhang P. (2017): Modified scaled boundary finite element analysis of 3D steady-state heat conduction in anisotropic layered media. - International Journal of Heat and Mass Transfer, vol.108, pp.2462-2471.

[35] Nowacki W. (1975): Dynamical Problems of Thermoelasticity. - Noordhoff, Leyden, The Netherlands.

[36] Vashisth A.K., Rani K. and Singh K. (2015): Quasi-static planar deformation in a medium composed of elastic and thermoelastic solid half spaces due to seismic sources in an elastic solid. - Acta Geophysica, vol.63, No.3, pp.605-633.

[37] Sneddon I.N. (1951): Fourier Transforms. - New York: McGraw-Hill.

[38] Garg N.R. and Singh S.J. (1985): Residual response of a multilayered half-space to two-dimensional surface loads. - Bull. Ind. Soc. Earthq. Tech., vol.22, pp.39-52.

[39] Ahrens T.J. (1995): Mineral physics and crystallography: a Handbook of physical constants. - American Geophysical Union, Washington, D.C.

[40] Aki K. and Richards P.G. (1980): Quantitative Seismology: Theory and Methods, Volumes I and II. - W.H. Freeman and Company, San Francisco, U.S.A.

[41] Schapery R.A. (1962): Approximate methods of transform inversion for viscoelastic stress analysis. - Proc. 4th US Nat. Congr. Appl. Mech., vol.2, pp.1075-1085.

Received: January 8, 2019

Revised: November 28, 2019 